\title{
NEW APPROACH TO METAL BIOMARKER DETECTION USING NONIONIZING ELECTROMAGNETIC FIELD
}

\begin{abstract}
Medical detection techniques used for preoperative scanning of certain marked areas are mostly based on ionizing radiation as it is in case of CT. This paper provides an unusual approach to detection of metal biomarkers implanted into the human body, based on nonionizing radiation and discusses problems and complications connected with chosen technique.
\end{abstract}

Keywords: Non-destructive evaluation; eddy current testing, metal biomarker detection, modeling and numerical simulation.

\section{Introduction}

The development in medicine and material engineering produced a group of materials called biomarkers which are used to highlight a specific part of biological object, mainly during diagnostic processes. This group consists of materials such as special dyes, plastics or metals. One could manage to write a book which would deal with the whole group of biomarkers, but the aim of this work is only the group of small metal biomarkers and their detection.

Metal biomarkers with a size of a few $\mathrm{mm}$ are often implanted into soft tissue of oncological patients to indicate the treated area of a tumor during the radiotherapy, and to observe further development of the disease in this area, as it is mentioned in [1]. That's why their correct detection is very important.

These biomarkers could be easily detected via commonly used medical detection techniques such as computer tomography (CT) or X-ray imaging, as it was published in [1,2 and 3]. The only disadvantage of these methods is that they are based on ionizing radiation and thus are harmful for human organism. An alternative for the detection could be provided by ultrasound. But the ultrasound probes are principally designed to focus on soft tissues, and need not resolve problems with detection of biomarker implanted into the bone. That's the reason for investigation of alternative detection methods.

\section{Materials and methods}

The aim of this work is to present some innovative ideas for the detection of small metal biomarkers. The first step of this investigation is selection of proper detection method which should not be anyway invasive or potentially harmful. According to research currently being pursued at the Department of electromagnetic and biomedical engineering at the University of Zilina, the most likely method to begin with is eddy current testing (ECT). This technique is not common in medical environment, but under certain conditions, it could principally help to solve some of the medical problems.

a. Eddy current testing principle

Non-destructive evaluation (NDE) is recently a very progressive industrial method used for determination of surface and subsurface defects, leaks, discontinuities, thermal anomalies, etc. ECT represents one branch of NDE. Eddy current testing is widely used for quality assurance of structural components made of conducting materials [4]. Its principle comes out from the electromagnetic induction phenomena. When a probe is electromagnetically linked with a part under inspection, the alternating magnetic field created by the probe driven with alternating current induces eddy currents in the test part [5]. Discontinuities or property variations in the test part change the flow pattern of the eddy currents and this change can be detected by the probe. The simple scheme of the ECT method principle is shown in Fig. 1.

\footnotetext{
* ${ }^{1}$ Roman Radil, ${ }^{1}$ Jan Barabas, ${ }^{2}$ Patrik Kamencay

${ }^{1}$ Department of Electromagnetic and Biomedical Engineering, Faculty of Electrical Engineering, University of Zilina, Slovakia

${ }^{2}$ Department of Telecommunications and Multimedia, Faculty of Electrical Engineering, University of Zilina, Slovakia

E-mail: roman.radil@fel.uniza.sk
} 


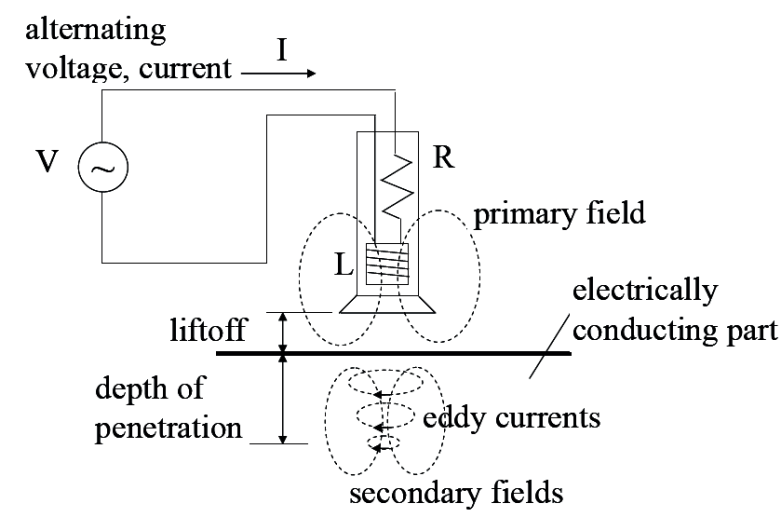

Fig.1 Eddy current testing principle [2]

\section{b. Biomarker selection}

It is well known that all implantable materials, not only biomarkers, must fulfill certain specific conditions of biocompatibility, which means that not all of the metal materials could be used as biomarkers. A very frequent solution to this problem in medicine is utilization of noble metals, especially gold. For this reason a gold biomarker shown in Fig. 2, called BiomarC, produced by the Aptum company, was selected.

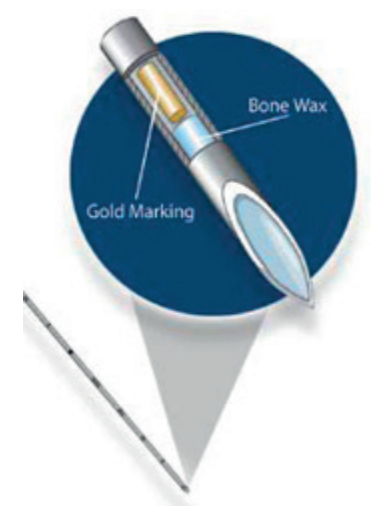

Fig. 2 BiomarC - gold biomarker at a size of $1.2 \times 3 \mathrm{~mm}$ located in the needle tip

Since gold represents a perfect conductive material, in comparison with human bone, the principle of ECT detection method could produce significant results. To verify the application of ECT method, the means of numerical simulations were used.

\section{Biological tissue modeling}

Biological environment, in which the biomarker should be detected, is a multilayer environment which could be generally divided into four layers - tissues: skin, fat, muscle and bone. Each of these tissues is characterized by its own dielectric properties which are frequency dependent. The frequency dependence of biological tissues in correlation with applied electromagnetic field (EMF) has been proven by many research works, some of which could be found in [6]. Tissue dielectric properties could be in general characterized by their relative permittivity:

$\varepsilon_{r e l}=\varepsilon^{\prime}-i \varepsilon^{\prime \prime}$

where $\varepsilon^{\prime}$ is relative permittivity of material, and $\varepsilon^{\prime \prime}$ is the outphase factor which can be computed as:

$\varepsilon^{\prime \prime}=\frac{\kappa}{\varepsilon_{0} \omega^{\prime}}$

where $\kappa$ is the total conductivity of material which also may contain a frequency-independent ionic contribution, $\boldsymbol{\varepsilon}_{0}$ is vacuum permittivity and $\omega$ is angular velocity.

With respect to the tissue dielectric properties in a wide frequency range, a high resolution model of human body has been created by authors in several research works [7, 8 and 9].

From the aforementioned, it is clear that the biological modeling should reflect the environment inhomogeneity and variability of the dielectric properties. According to this, two modeling phantoms could be basically defined:

- tomographic phantom,

- stylized phantom.

"Tomographic phantoms are obtained from modern medical imaging techniquesas the Magnetic Resonance Imaging (MRI), or the CT and represent the body by sampled volume pixels, the so-called voxels.

Stylized phantoms describe the human anatomy by using simple mathematical equations of analytic geometry" [6].

\section{Experimental section}

As the primary problem of this work is the biomarker detection, the problem with the modeling of biological tissues was simplified, thus the stylized phantom approach was deemed as sufficient. Furthermore, the region of the gold biomarker insertion was consulted with medical experts, and the area of pelvis where the thickness of tissue is only few mm, was chosen.

a. Modeling and numerical simulations

The decisions reduce the number of modeled layers to three - skin, subcutaneous fat and bone. So the model of biological environment could be created as shown in Fig. 3 . 


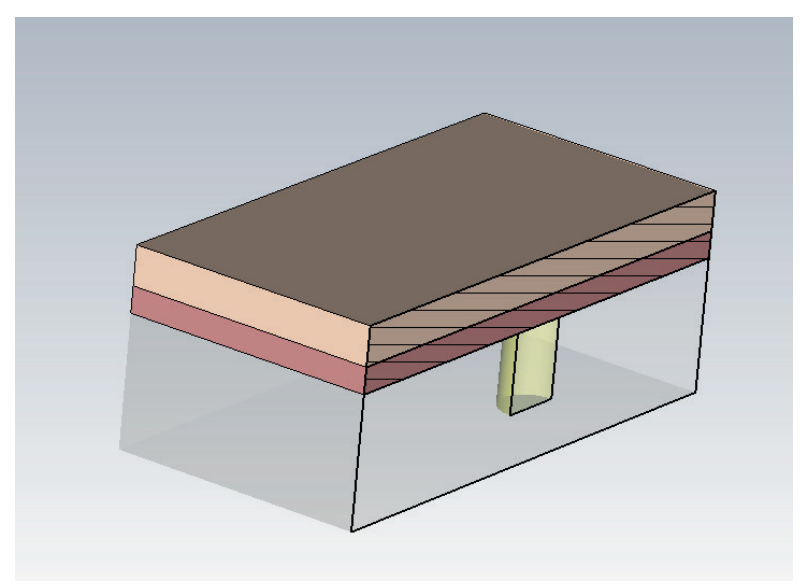

Fig. 3 Model of biological tissues with implanted gold biomarker - crossection

The dielectric properties of modeled tissues were changed according to the frequency of intended EMF application. An example of the fat tissue dielectric properties at a chosen frequency range is presented in Table 1.

Fat tissue dielectric properties [7, 8 and 9]

Table 1

\begin{tabular}{|c|c|c|c|}
\hline $\begin{array}{c}\text { Frequency } \\
{[\mathrm{kHz}]}\end{array}$ & $\begin{array}{c}\text { Conductivity } \\
{[\mathbf{S} / \mathrm{m}]}\end{array}$ & $\begin{array}{c}\text { Relative } \\
\text { permittivity }\end{array}$ & $\begin{array}{c}\text { Penetration } \\
\text { depth }[\mathrm{m}]\end{array}$ \\
\hline 5 & 0.023589 & 2816.5 & 47.118 \\
\hline 9.5 & 0.023815 & 1161.4 & 33.894 \\
\hline 14 & 0.023926 & 705.36 & 27.817 \\
\hline 18.5 & 0.023999 & 502.77 & 24.144 \\
\hline 23 & 0.024055 & 390.51 & 21.62 \\
\hline 27.5 & 0.0241 & 319.83 & 19.749 \\
\hline 32 & 0.024137 & 271.47 & 18.291 \\
\hline 36.5 & 0.024169 & 236.41 & 17.114 \\
\hline 41 & 0.024198 & 209.87 & 16.137 \\
\hline 45.5 & 0.024223 & 189.1 & 15.31 \\
\hline 50 & 0.024246 & 172.42 & 14.598 \\
\hline
\end{tabular}

The data in Table 1 are computed using an application based on the parametric model for the calculation of the dielectric properties of body tissues developed by C. Gabriel et al. [7, 8 and 9]. The given frequency range was selected to be possibly verified via real measurements using the Rohmann GmbH ELOTEST B300 - a commercial device for ECT which works at $10 \mathrm{~Hz}-10$ $\mathrm{MHz}$ frequency range.

Before starting with the simulation an ECT probe has to be modeled. The probe was designed as a small copper wire coil, driven by 0.1 ampere sinusoidal current, wound around a ferrite core. To avoid noise produced by surrounding appliances, an iron casing was designed. The simplified scheme of the model is displayed in Fig. 4 together with the probe proportions.

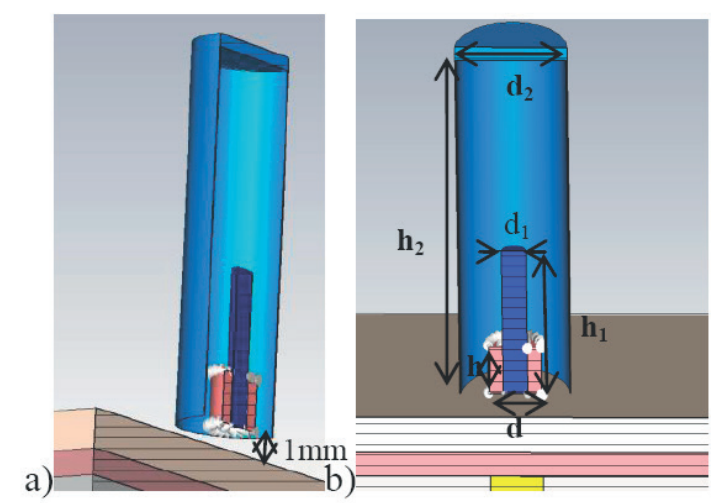

Fig. 4 Cross-section of probe modeling: a) location above the skin, b) probe parameters: external coil diameter $d=2.2 \mathrm{~mm}$, winding height $h=$ $2 \mathrm{~mm}$, core diameter $d_{1}=1.1 \mathrm{~mm}$, core height $h_{1}=6 \mathrm{~mm}$, diameter of the cover $d_{2}=5 \mathrm{~mm}$, cover height $h_{2}=14.5 \mathrm{~mm}$

Once the model design is completed, the numerical simulations take place. Since the metal biomarker could be considered as an electrically small structure, the low frequency domain solver was chosen to conduct the computations. The solver is based on finite integration technique (FIT), which represents the discretization of each Maxwell equation. An example of grid creation using FIT is shown on Fig.5.

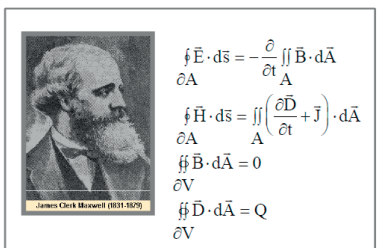

Maxwell's equations

$$
\text { div curl }=0
$$$$
\text { curl grad }=0
$$

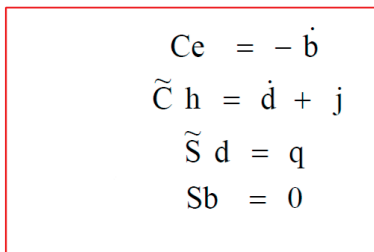

Grid equations

\begin{tabular}{|c|}
$S C=0$ \\
$\widetilde{C} S=0$
\end{tabular}

Fig. 5 Discretizing Maxwell's equations to create a grid

The choice of hexahedral mesh was essential to achieve the best geometry approximation available. Four simulation frequencies from the given frequency range were chosen to be calculated with. The said frequencies are 5, 10, 25 and $50 \mathrm{kHz}$. Results of the experiments with the gold biomarker detection using simulations can be seen as 3-D capture of electrical intensity field (E-field) in Fig. 6 where results of chosen calculation frequencies are compared. 

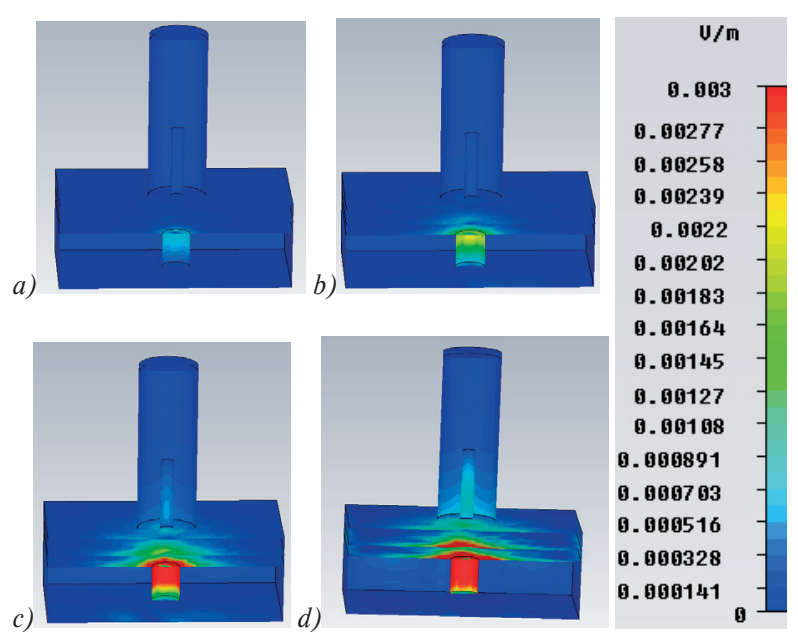

Fig. 6 Magnetoquasistatic simulation results - E-field at frequency: a) $5 \mathrm{kHz}$, b) $10 \mathrm{kHz}$, c) $25 \mathrm{kHz}$ d) $50 \mathrm{kHz}$.

As can be seen from the color map of results, the higher the applied frequency is, the better the detection of the biomarker should be. But, on the other hand, the capture of E-field distribution should be considered in accordance with the absorption law theory and standards presented in [10]. This means the energy losses in soft tissues (especially skin) should be expected at higher frequencies, and for this reason it seems that the smaller frequency values up to $25 \mathrm{kHz}$ could be optimal for the detection process.

\section{b. Experimental verification of numerical simulations}

Obtained simulation results showed a possibility of the biomarker detection using the ECT method. To verify the results, experiments with real measurements on 3D phantom model of human pelvis presented in Fig. 7, were collected.

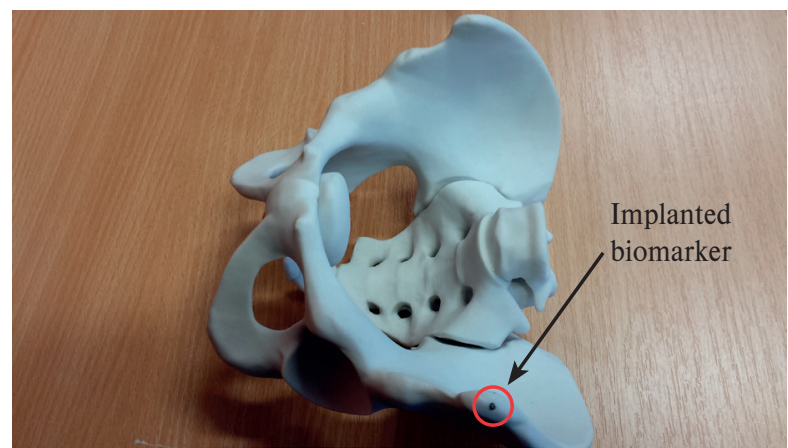

Fig. 7 3D phantom model of human pelvis with implanted biomarker

The ECT device ELOTEST B300, mentioned before in this article was chosen to be used for the real measurements. Also a special probe, proportionally comparable with the one modeled during the simulations, was used for eddy current excitation and detection. Both devices are depicted in Fig. 8.
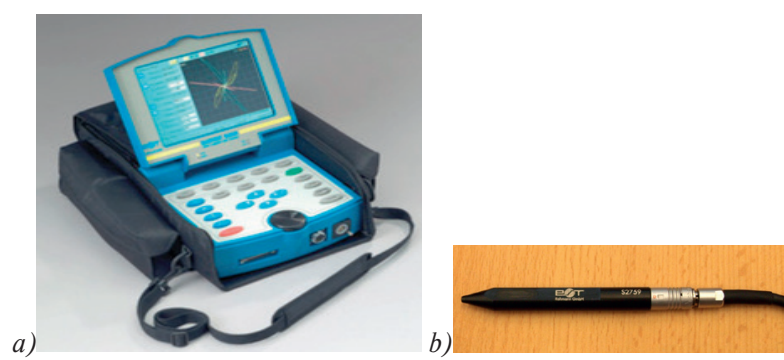

Fig. 8 ECT devices: a)Rohmann GmbH ELOTEST B300, b) ECT probe

Since there was no other material available to simulate at least a skin tissue, it was opted to measure each of the simulation frequencies at various distances from the pelvis surface to verify whether or not such a small gold particle could be detected. The detection process results show interesting contrast between numerical simulations and real measurements, which is documented by Table 2 . The successful detections are marked as "1", undetected cases are marked as "-".

Results of measurements

Table 2

\begin{tabular}{|c|c|c|c|c|}
\hline \multirow{2}{*}{$\begin{array}{c}\text { Distance } \\
\text { from surface } \\
{[\mathrm{mm}]}\end{array}$} & \multicolumn{4}{|c|}{ Frequency } \\
\hline & $1 \mathrm{kHz}$ & $10 \mathrm{kHz}$ & $25 \mathrm{kHz}$ & $50 \mathrm{kHz}$ \\
\hline 0.5 & - & 1 & 1 & 1 \\
\hline 1 & - & - & 1 & 1 \\
\hline 3 & - & - & - & 1 \\
\hline 5 & - & - & - & - \\
\hline
\end{tabular}

To enhance the experimental verification of the numerical simulations, two more configurations were tested for the biomarker detection. The first one was the probe using a giant magneto-resistive (GMR) sensor as a detector while the second one uses an anisotropic magneto-resistive sensor (AMR) for detection. The function of both the GMR and AMR sensors is principally described in [11]. In our measurements two excitation coils were used to create the magnetic field which should change due to the presence of the biomarker, and these changes should be detected by the sensor. The whole probe composition is shown in Fig. 9, and results for each sensor are presented in Table 3.

The failure of the detection process led to only one conclusion - magneto-resistive sensors couldn't be used as detectors of the gold biomarkers. Furthermore, from the presented results it is clear that real life measurements uncovered new problems. On the one hand, it was confirmed to use the highest possible frequency (in case of ELOTEST), but, on the other hand, it was shown that the distance between the probe and detected biomarker should be very small as the last detected case was at frequency of $50 \mathrm{kHz}$ in the distance of $2.5 \mathrm{~mm}$. The detection distance is quite a big problem, especially when the influence of other biological tissues wasn't considered during the experiments. Another problem is supposed to be with the material magnetic properties of the used 


\begin{tabular}{|c|c|c|c|c|c|c|c|c|}
\hline \multirow{2}{*}{ Distance from surface [mm] } & \multicolumn{4}{|c|}{ Frequency AMR } & \multicolumn{4}{|c|}{ Frequency GMR } \\
\hline & $1 \mathrm{kHz}$ & $10 \mathrm{kHz}$ & $25 \mathrm{kHz}$ & $50 \mathrm{kHz}$ & $1 \mathrm{kHz}$ & $10 \mathrm{kHz}$ & $25 \mathrm{kHz}$ & $50 \mathrm{kHz}$ \\
\hline 0.5 & - & - & - & 1 & - & - & - & - \\
\hline 1 & - & - & - & - & - & - & - & - \\
\hline 3 & - & - & - & - & - & - & - & - \\
\hline 5 & - & - & - & - & - & - & - & - \\
\hline
\end{tabular}

biomarker and its small dimensions. All mentioned problems could be considered as reasons for detection failure.

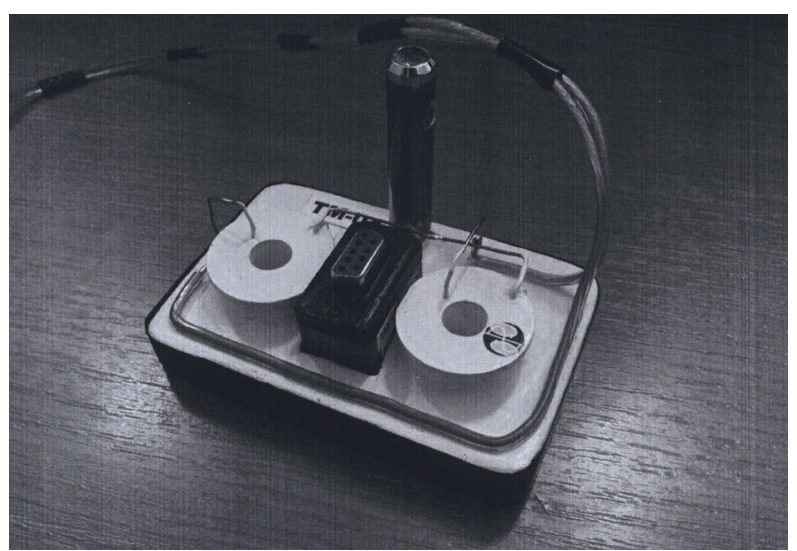

a)

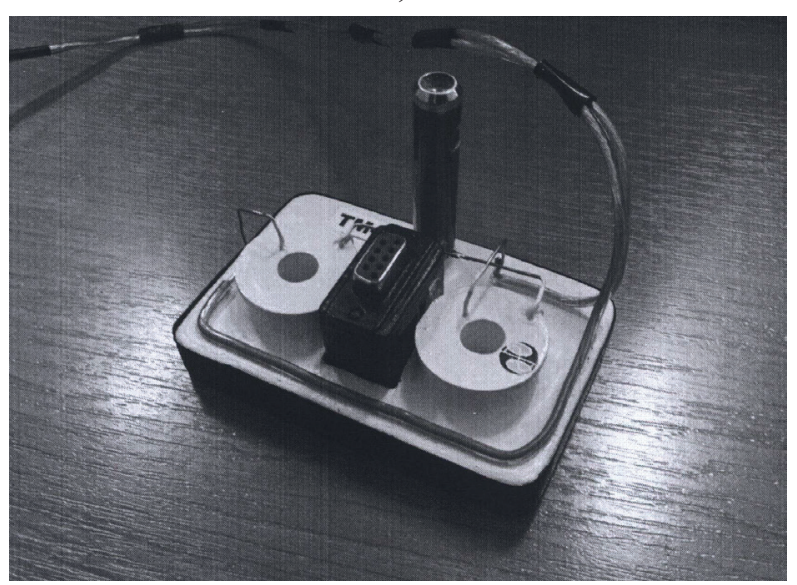

b)

Fig. 9 Composition of the detection probe using: a) AMR sensor b) GMR sensor

A. Research focused on problem solving

To address the observed problems further experiments were conducted regarding the ECT method. First of all, a new probe model was created. The probe was designed as a $3 \times 3$ matrix of previously created single coil sensors. The value of the driving current remains the same, with the exception of central coil which was designed as a detection coil to measure the changes caused by the presence of the biomarker in the tissue. The whole configuration as well as results of numerical simulations is illustrated in Fig. 10.

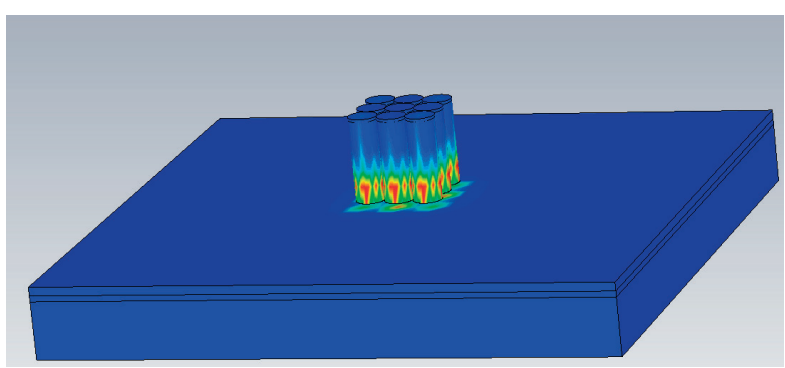

a)

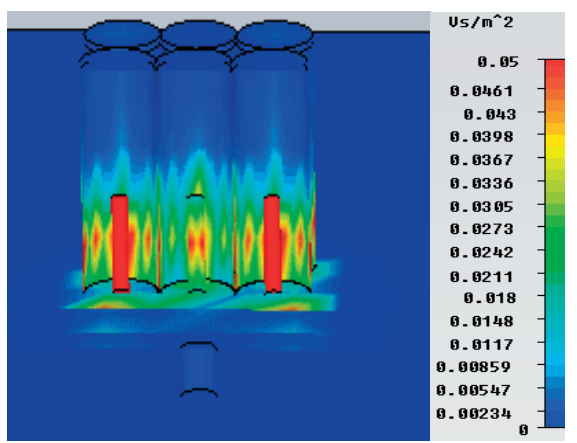

b)

Fig. 10 Simulation results: a) Matrix configuration of sensors, b) cross-section with induction changes on central detector

To verify this possibility of detection a matrix of GMR sensors, shown in Fig. 11 was used for measurements.

But as it is clear from the table, attached to the capture in Fig. 11 , the only detected case was at the frequency of $100 \mathrm{kHz}$, so no progress was achieved. 


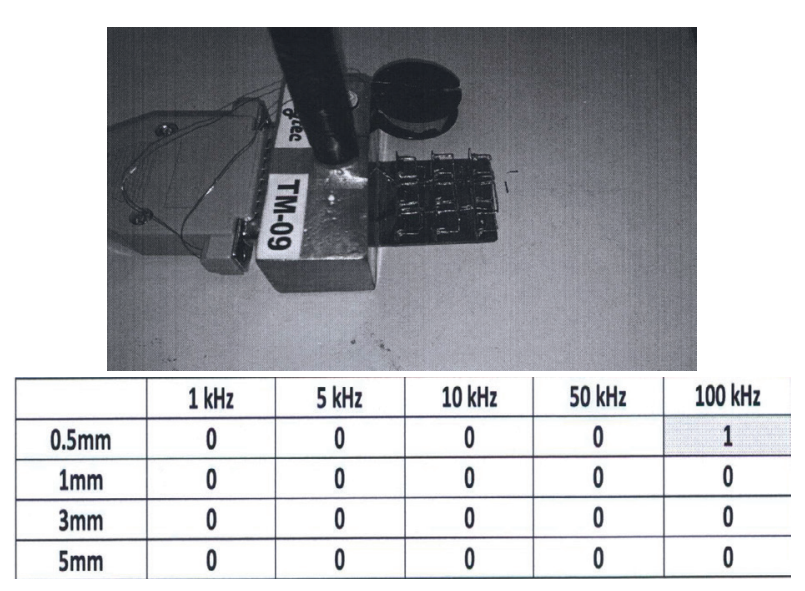

Fig. $113 \times 3$ matrix of GMR sensors with detection results

\section{Conclusion}

According to the aim of presented work, an innovative approach to small metal biomarker detection using the ECT method was presented. Numerical simulation results uncover potential of the proposed method. Its main advantages include fast examination rate, non-invasiveness and cheapness.
But experiments with real measurements depict another picture and showed limits of the detection method. Probably the most important limitations identified as the main reasons of detection failure are: the distance between probe and biomarker, the biomarker dimensions and biomarker material.

As the biomarker dimensions must stay as low as possible, to solve the mentioned problems the biomarker material could be changed from gold to iron and thus better magnetic properties to strengthen the detection process can be obtained and obviously the detection distance can be enlarged. Of course, it cannot be forgotten that the biocompatibility must be assured in order of iron biomarker implementation.

The mentioned solution proposal together with other detection possibilities are currently investigated and results of this research will be published soon.

\section{Acknowledgement}

This work has been supported by the European Regional Development Fund and the Ministry of Education of the Slovak Republic, ITMS 26220220153 - Centre of competence for research and development in the area of diagnostics and therapy of oncological diseases.

\section{References}

[1] DEHNAD, H., NEDERVEEN, A.J., VAN DER HEIDE, U.A., VAN MORSELAAR, R.J.A., HOFMAN, P., LAGENDIJK, J.J.W.: Clinical Feasibility Study for the Use of Implanted Gold Seeds in the Prostate as Reliable Positioning Markers During Megavoltage Irradiation, Radiotherapy and Oncology, vol. 55, 2003, pp. 295-302

[2] KITAMURA, K., SHIRATO, H., SHIMIZU, S., SHINOHARA,N., HARABAYASHI, T., SHIMIZU, T., KODAMA, Y., ENDO, H., ONIMARU, R., NISHIOKA, S., AOYAMA, H., TSUCHIYA, K., MIYASAKA, K.: Registration Accuracy and Possible Migration of Internal Fiducial Gold Marker Implanted in Prostate and Liver Treated with Real-time Tumor-tracking Radiation Therapy (RTRT), Radiotherapy and Oncology, vol. 62, 2002, pp. 275-281

[3] KOTTE, A.N.T.J., HOFMAN,P., LAGENDIJK, J.J.W., VAN VULPEN, M., VAN DER HEIDE, U.A.: Intrafraction Motion of the Prostate During External-beam Radiation Therapy: Analysis of 427 patients with implanted fiducial markers, Int. J. Radiation Oncology Biol. Phys., vol. 69, No. 2, 2007, pp. 419 - 425

[4] JANOUSEK, L., CAPOVA, K., GOMBARSKA, D., SMETANA, M.: Progress in Eddy-current Non-destructive Evaluation of Conductive Materials, Acta Technica CSAV, vol. 55, No. 1, 2010, pp. 13-28.

[5] STRAPACOVA, T., SMETANA, M., CAPOVA, K.: Non-destructive Investigation of the Artificial Heart Valves Using Eddy Current Testing - an Inovative Approach, Lecture Notes in Computer Science, vol. 7339, 2012, pp. 331-340.

[6] BARCHANSKI, A.: Simulations of Low-Frequency Electromagnetic Fields in the Human Body, Dissertation thesis. Darmstadt: Technische Universitat, 2007, pp.1-111

[7] GABRIEL, S., CORThOUT, E., GABRIEL, C.: The Dielectric Properties of Biological Tissues: I. Literature Survey. Physics in Medicine and Biology, vol. 41, No. 11, 1996, pp. 2231-2249. ISSN: 1361-6560

[8] GABRIEL, S., LAU, R. W., GABRIEL, C.: The Dielectric Properties of Biological Tissues: II. Measurements in the Frequency Range $10 \mathrm{~Hz}$ to $20 \mathrm{GHz}$, Physics in Medicine and Biology, vol. 41, No.11, 1996, pp. 2251-2269, ISSN: 1361-6560

[9] GABRIEL, S., LAU, R. W., GABRIEL, C.: The Dielectric Properties of Biological Tissues: III. Parametric Models for the Dielectric Spectrum of Tissues, Physics in Medicine and Biology, vol. 41, No. 11,1996, pp. 2271-2293. ISSN: 1361-6560 
[10] MICHAELSON, S. M.: Human Exposure to Nonionizing Radiant Energy-Potential Hazards and Safety Standards, Proc. of the IEEE, vol. 60, No. 4, 1972, pp. 389-421. ISSN: 0018-9219G.

[11] JANOUSEK, L., REBICAN, M. I., SMETANA, M., STRAPACOVA, T., DUCA, A., PERNISOVA, V.: Recent Innovative Solutions in Eddy Current Non-destructive Diagnosis, Communications - Scientific Letters of the University of Zilina, vol. 15, No. 2, 2013, pp. 102-108. ISSN 1335-4205. 